\title{
Impulskontrollstörungen in der ICD-11
}

\author{
Susanne Bründl ${ }^{1}$ iD $\cdot$ Johannes Fuss ${ }^{1}$ \\ Eingegangen: 9. Dezember 2020 / Angenommen: 15. Dezember 2020 / Online publiziert: 8. Januar 2021 \\ (c) Der/die Autor(en) 2021
}

\section{Zusammenfassung}

Dieser Beitrag beschäftigt sich mit der Neuerung der Diagnoserichtlinien in der 11. Revision der International Classification of Diseases and Related Health Problems (ICD-11) im Kapitel der Impulskontrollstörungen. Die Diagnosen Pyromanie und Kleptomanie bleiben nahezu unverändert erhalten. Die Diagnose pathologisches Glücksspiel verliert ihren Status als Impulskontrollstörung und wird Teil des neuen Kapitels der Verhaltenssüchte. Neu im Kapitel der Impulskontrollstörungen ist die Wiederaufnahme der Diagnose intermittierende explosible Störung sowie die neue, kontrovers diskutierte Diagnose zwanghafte sexuelle Verhaltensstörung. Der Artikel stellt Änderungen der Diagnosen zwischen der ICD-11 und ihrer Vorgängerversion (ICD-10) gegenüber, greift aktuelle Diskussionen rund um das Kapitel der Impulskontrollstörungen auf und beschreibt klinische sowie forensisch relevante Implikationen der modifizierten und neu eingeführten Diagnosen.

Schlüsselwörter Zwanghafte sexuelle Verhaltensstörung $\cdot$ Hypersexualität $\cdot$ Pyromanie $\cdot$ Kleptomanie $\cdot$ Intermittierende explosible Störung

\section{Impulse control disorders in the ICD-11}

\begin{abstract}
This article deals with the new diagnostic guidelines in the 11th revision of the International Classification of Diseases and Related Health Problems (ICD-11) in the chapter on Impulse Control Disorders. The diagnoses pyromania and kleptomania remain almost unchanged. The diagnosis of Pathological Gambling loses its status as Impulse Control Disorder and becomes part of the new chapter of Behavioral Addictions. New in the chapter on Impulse Control Disorders is the resumption of the diagnosis Intermittent Explosive Disorder and the new, controversially discussed diagnosis Compulsive Sexual Behavior Disorder. The article compares changes in diagnostic guidelines between the ICD-11 and its predecessor version (ICD-10), picks up on current discussions surrounding the chapter on Impulse Control Disorders, and describes clinically and forensically relevant implications of the modified and newly introduced diagnoses.
\end{abstract}

Keywords Compulsive Sexual Behavior Disorder $\cdot$ Hypersexuality $\cdot$ Pyromania $\cdot$ Kleptomania $\cdot$ Intermittent Explosive Disorder

Das Kapitel „Impulskontrollstörungen“ im 6. Kapitel der 11. Revision der International Classification of Diseases and Related Health Problems (ICD-11; https://icd.who.int/ dev11/l-m/en) löst das Kapitel F63 „Abnorme Gewohnhei-

PD Dr. med. Johannes Fuss

jo.fuss@uke.de

1 Institut für Sexualforschung, Sexualmedizin und Forensische Psychiatrie, Universitätsklinikum Hamburg-Eppendorf, Martinistr. 52, 20246 Hamburg, Deutschland ten und Störungen der Impulskontrolle“ aus der vorherigen Version (ICD-10; Dilling et al. 1991) ab. Das korrespondierende Kapitel in der neusten Revision des Diagnostic and Statistical Manual of Mental Disorders (DSM-5; American Psychiatric Association 2013) sind „Disruptive, Impulskontroll- und Sozialverhaltensstörungen“, ehemals als „Störungen der Impulskontrolle, nicht andernorts klassifiziert" bezeichnet (DSM-III und -IV). Während die Impulskontrollstörungen in der ICD-10 durch die künstliche Beschränkung der Störungskapitel im Rahmen eines dezimalen Kodierungssystems (F0-F9) noch dem Kapitel F6 „Persönlich- 
keits- und Verhaltensstörungen“" zugeordnet waren, stehen sie nun als alleinige Kategorie und umfassen folgende Diagnosen:

- Pyromanie (pathologische Brandstiftung; ICD-11: 6C70) - ,pyromania“,

- Kleptomanie (pathologisches Stehlen; ICD-11: 6C71) „kleptomania“,

- zwanghafte sexuelle Verhaltensstörung ${ }^{1}$ (ICD-11: 6C72) - „,compulsive sexual behavior disorder“,

- intermittierende explosible Störung (ICD-11: 6C73) ,,intermittent explosive disorder“".

In der aktuellen digitalen Version der ICD-11 (https:// icd.who.int/dev11/1-m/en), die im Januar 2022 in Kraft treten soll, finden sich zusätzlich Querverweise zu den ehemals im Kapitel F63 enthaltenen Diagnosen pathologisches Glückspiel (,gambling disorder") und Trichotillomanie sowie zur neuen Diagnose pathologisches Spielen (,gaming disorder"). pathologisches Glücksspiel (6C50) und pathologisches Spielen (6C51) bilden in der ICD-11 die neue Kategorie der Verhaltenssüchte. Trichotillomanie (6B25.0) wird zukünftig den Zwangsstörungen zugeordnet. Die neu geordnete Gruppe der Impulskontrollstörungen soll nachfolgend hinsichtlich ihrer Veränderungen gegenüber der ICD-10 beschrieben und einschließlich ihrer Implikationen diskutiert werden.

Impulskontrollstörungen sind in der ICD-11 gekennzeichnet durch wiederholtes Versagen, einem starken Impuls, Antrieb oder Drang zu widerstehen, eine Handlung auszuführen, die kurzfristig belohnend ist, längerfristig jedoch mit negativen Folgen für die Person selbst oder andere, starkem Leidensdruck oder erheblichen Beeinträchtigungen in persönlichen, familiären, sozialen, schulischen, beruflichen oder anderen wichtigen Funktionsbereichen verbunden ist.

Verhaltensweisen (Brandstiftung, Diebstahl, sexuelles Verhalten und explosive Ausbrüche) im Zusammenhang mit Störungen der Impulskontrolle gehen typischerweise mit steigender Anspannung oder affektiver Erregung vor dem Verhalten oder bei Widerstand gegen das Verhalten einher, sowie mit Vergnügen, Nervenkitzel, Befriedigung oder Abnahme der Anspannung während oder nach dem Verhalten. Im Verlauf der Störung können das Empfinden von Anspannung oder Erregung vor dem Verhalten sowie die damit einhergehende Befriedigung jedoch abnehmen. Hinzukommen können Gefühle von Schuld oder Scham nach Ausführen des Verhaltens.

Damit wurde die Konzeptualisierung von Impulskontrollstörungen im Vergleich zur ICD-10 konkretisiert, in

\footnotetext{
${ }^{1}$ Im vorliegenden Artikel wird der Begriff „Zwanghafte Sexuelle Verhaltensstörung “ genutzt - eine offizielle Übersetzung des Begriffs Compulsive Sexual Behavior Disorder liegt aktuell noch nicht vor.
}

der sie lediglich als wiederholte impulsive Handlungen ohne klare rationale Motivation mit negativen Konsequenzen für Betroffene selbst oder andere Menschen definiert wurden. Die neue Definition relativiert den Gedanken, dass es sich bei den „Störungen der Impulskontrolle“ um unkontrollierbares Verhalten handelt, im Sinne kurzer und spontaner Handlungsimpulse, denen, ohne darüber nachzudenken, nachgegeben wird. Dadurch wird deutlicher, dass entgegen dem intuitiven Verständnis des Begriffs auch Verhaltensweisen inbegriffen sind, denen eine gewisse Absicht und Abwägung oder ein Wunsch nach Vergnügen und Nervenkitzel im Vorfeld zugrunde liegen. Vor allem bei Brandstiftung, Diebstahl oder sexuellem Verhalten ist davon auszugehen, dass oft eher bedacht und vorsätzlich vorgegangen wird als unüberlegt und impulsiv (Kröber 2015).

\section{Pyromanie (6C70) und Kleptomanie (6C71)}

Die Diagnosen Pyromanie und Kleptomanie, erstmals im DSM-III als Störungen der Impulskontrolle gelistet, sind seit über 30 Jahren im Grundsatz unverändert. Die ICD-11 betont lediglich in Abgrenzung zu normalem Verhalten das Fehlen eines nachvollziehbaren Motivs als pathologisches Kernmerkmal (z.B., dass die Güter nicht zur persönlichen Nutzung oder wegen ihres finanziellen Wertes gestohlen werden, oder dass der Brandstiftung kein nachvollziehbares Motiv wie z. B. Sabotage zugrunde liegt). Beide Störungen gehen auf das Konzept der Monomanien von Esquirol (1838) und Marc (1844) zurück, die sie als krankhaft gestörte Kontrolle über eine spezifische Handlung (Brandstiftung oder Stehlen) ohne weitere Zeichen einer psychischen Störung definierten (Leygraf 2009). In der Vergangenheit wurde kritisiert, dass Pyromanie (absichtsvolles Feuerlegen) und Kleptomanie (pathologisches Stehlen) in ihrer Operationalisierung eher im Sinne gesellschaftlichen Fehlverhaltens als im Sinne umschriebener psychischer Störungen anmuten (Kröber 2015). Kröber (2015) zog Parallelen zwischen dem für Impulskontrollstörungen typischen Anspannungszustand vor sowie Entspannungszustand nach der Handlung und anderen wichtigen sportlichen, beruflichen oder Alltagshandlungen (z.B. an einem Wettkampf teilnehmen). Damit stellte er die Konzeptualisierung von Pyromanie und Kleptomanie als psychische Störungen infrage. Auch empirische Untersuchungen zeigen, dass pathologische Brandstiftung oder pathologisches Stehlen selten isoliert, sondern vielmehr komorbide zu unterschiedlichen psychischen Störungen auftreten (Barnett 2005). So wurde empfohlen, die Verhaltensweisen eher als Symptome anderer psychischer Störungen und Belastungen zu verstehen (Leygraf 2009), da deren Betrachtung als eigenständige Störungsbilder eine dahinterliegende psychopathologische Problematik verdecken könnte. Dass die Störungen so gut wie 
nie isoliert vorliegen, unterstreichen auch die unbekannte Prävalenz von Pyromanie (Burton et al. 2012) und die niedrige Prävalenz von 0,3-0,6\% (Frauen: Männer, 3: 1) für Kleptomanie (Torales et al. 2020). Dementsprechend wurden beide Störungsbilder bis dato sowohl in der klinischen als auch in der Forschungspraxis, ebenso wie in den Überarbeitungsprozessen der ICD-11, vernachlässigt - womit nicht davon auszugehen ist, dass sich der Zustand im Zuge der aktuellen Revision verändern wird.

\section{Zwanghafte sexuelle Verhaltensstörung (6C72)}

Ein Phänomen, das erstmals vor über 100 Jahren beschrieben (von Krafft-Ebing 1903) und seither unter verschiedensten Labels - wie Hypersexualität, Sexsucht, sexuelle Impulsivität, sexuelle Zwanghaftigkeit (Fuss et al. 2019a) diskutiert und untersucht wurde (Briken 2016 für einen historischen Überblick), wird nun erstmals als operationalisierte psychische Störung in ein Klassifikationssystem eingehen. Ein früherer Vorschlag über die Aufnahme des Zustands als ,hypersexual disorder“ (Kafka 2010) in das DSM-5 (American Psychiatric Association 2013) wurde aufgrund unzureichender empirischer Datenlage sowie klinischer, sozialer und rechtlicher Bedenken abgelehnt (Kafka 2014). In der ICD-10 findet sich lediglich im Kapitel der sexuellen Funktionsstörungen die Diagnose gesteigertes sexuelles Verlangen (F52.7), jedoch ohne diagnostische Richtlinien oder Symptombeschreibungen. Die zwanghafte sexuelle Verhaltensstörung (Compulsive sexual behavior disorder) soll in der ICD-11 ins Kapitel der Impulskontrollstörungen eingeordnet und wie folgt definiert ${ }^{2}$ werden:

Ein anhaltendes Unvermögen, intensive, sich wiederholende sexuelle Impulse oder Triebe zu kontrollieren, was zu wiederholtem sexuellem Verhalten führt, das sich in einem oder mehreren der folgenden Punkte manifestiert:

- Die Ausübung der sexuellen Aktivitäten hat zentralen Stellenwert im Leben einer Person erlangt. Andere Interessen, Aktivitäten und Pflichten sowie die persönliche Fürsorge und Gesundheit werden aufgrund der Aktivitäten vernachlässigt.

- Die Person hat zahlreiche erfolglose Versuche unternommen, das Sexualverhalten zu kontrollieren oder deutlich zu reduzieren.

\footnotetext{
${ }^{2}$ Hierbei handelt es sich um unsere Übersetzung der diagnostischen Richtlinien - eine offizielle Übersetzung der Diagnosekriterien liegt aktuell noch nicht vor.
}

- Die Person führt das repetitive Sexualverhalten trotz nachteiliger Folgen (z. B. wiederholte Beziehungsbrüche, berufliche Konsequenzen, negative Auswirkungen auf die Gesundheit) fort.

- Die Person führt das repetitive Sexualverhalten fort, auch wenn sie wenig oder keine Befriedigung daraus zieht.

Neben den Merkmalen der exzessiven Sexualität ist für die nosologische Einordnung entscheidend, dass das abweichende Sexualverhalten nicht nur im Rahmen besonderer Lebensumstände (z. B. nach einer Trennung) vorübergehend auftritt, sondern über einen längeren Zeitraum (z.B. mindestens 6 Monate) besteht. Außerdem sind für den Störungscharakter ähnlich wie bei der Konzeptualisierung anderer Störungsbilder ausgeprägter Leidensdruck oder Beeinträchtigungen in wichtigen Lebensbereichen notwendig. Leidensdruck hingegen, der ausschließlich durch moralische Wertungen oder die Missbilligung besonderer sexueller Verhaltensweisen entsteht, schließt die Diagnose einer zwanghaften sexuellen Verhaltensstörung aus. Beispielhaft sei an eine religiöse Person zu denken, die der Überzeugung ist, dass Masturbation und das Betrachten von Pornografie eine Sünde darstellen. Da es sich um ein breit gefasstes Konstrukt mit unterschiedlichen Ausprägungen und Wirkmechanismen handelt, sind die Diagnosekriterien relativ allgemein gefasst und vermeiden eine Konzentration auf mögliche Ätiologie (z.B. traumatische sexuelle Erfahrungen) oder Kontexte, in denen die sexuellen Handlungen auftreten können (z.B. als Bewältigungsstrategie für negative Emotionen) - dies steht im Gegensatz zur abgelehnten Konzeptualisierung für die DSM-5 (Stein et al. 2020).

In der Praxis werden von hilfesuchenden Personen am häufigsten Verhaltensweisen wie exzessive Masturbation und Nutzung von Pornografie berichtet, wobei sich das heterogene Krankheitsbild auch in interaktiven sexuellen Aktivitäten, z. B. Internet-, Telefonsex oder ungeschütztem Geschlechtsverkehr mit häufig wechselnden Sexualpartnern, äußern kann (Mead und Sharpe 2018). Über die Verbreitung in der Bevölkerung konnten aufgrund des bisherigen Fehlens offiziell anerkannter Diagnosekriterien und validierter Diagnoseinstrumente wenig zuverlässige Aussagen getroffen werden. Ältere epidemiologische Studien zu verwandten Konstrukten schätzen, dass 5-6\% der Allgemeinbevölkerung von dieser Symptomatik betroffen sein könnten (Carnes 1991; Coleman 1991). In einer landesweit repräsentativen Stichprobe aus den USA wiesen 10,3\% der Männer und 7,0\% der Frauen klinisch relevante Beeinträchtigungen in Verbindung mit Schwierigkeiten bei der Kontrolle sexueller Gefühle, Triebe und Verhaltensweisen auf (Dickenson et al. 2018). Wahrscheinlich handelt es sich dabei aber um eine Überschätzung der realen Prävalenz (Klein et al. 2014). Inwieweit es sich um ein zunehmendes Syndrom handelt, dass möglicherweise durch die Omniprä- 
senz, leichte und anonyme Verfügbarkeit sexueller Inhalte im Internet begünstigt wird (Griffiths 2012), wird sich in zukünftigen longitudinalen Studien auf Basis offizieller Diagnosekriterien zeigen.

\section{Intermittierende explosible Störung (6C73)}

Mit der intermittierenden explosiblen Störung zieht eine weitere Diagnose in das Manual der ICD-11 ein, die in der ICD-10 nicht enthalten ist. Im Gegensatz zur zwanghaften sexuellen Verhaltensstörung existierten aber bereits zuvor Diagnosekriterien. Die intermittierende explosible Störung geht aus früheren DSM- und ICD-Diagnosen zu Persönlichkeitsstörungen (aggressiver Typ) hervor und ist sowohl in DSM-III bis DSM-5 als auch bereits in der ICD9-CM (1979) enthalten. In der ICD-10 wurde die Diagnose zugunsten der emotional instabilen Persönlichkeitsstörung (impulsiver Typ) nicht gelistet (Werdenich und Padlesak 2006). Nachdem die in früheren DSM-Auflagen relativ breit gefassten und vage gehaltenen Diagnosekriterien im Rahmen der 5. DSM-Revision expliziert wurden, soll in Anlehnung daran für die ICD-11 folgende Definition ${ }^{3}$ der intermittierenden explosiblen Störung gelten:

- wiederholte kurze Episoden verbaler oder physischer Aggression oder Zerstörung von Eigentum, die ein Versagen bei der Kontrolle aggressiver Impulse darstellen,

- wobei die Intensität des Ausbruchs oder der Grad der Aggressivität in erheblichem Missverhältnis zur Provokation oder zu den auslösenden psychosozialen Stressfaktoren steht.

- Die Symptome lassen sich nicht besser durch eine andere psychische, verhaltensbedingte oder neurologische Störung erklären und sind nicht Teil eines Musters chronischer Wut und Reizbarkeit (z. B. bei der oppositionellen Trotzstörung).

- Das Verhaltensmuster ist von ausreichender Schwere, um zu einer signifikanten Beeinträchtigung in persönlichen, familiären, sozialen, schulischen, beruflichen oder anderen wichtigen Funktionsbereichen zu führen.

Das Auftreten anschließender Schuldgefühle gemäß Diagnoserichtlinien der ICD-9-CM fehlt im DSM-System und wird in der ICD-11 ebenfalls fallen gelassen. Anders als das DSM-5 verzichtet die ICD-11 auf eine Quantifizierung der impulsiven Ausbrüche oder Kontrollverluste. Die Diagnose kann vergeben werden, wenn das Verhalten über einen längeren Zeitraum (z.B. mindestens 6 Monate) regelmäBig auftritt. Es handelt sich also um ein anhaltendes Mus-

\footnotetext{
${ }^{3}$ Hierbei handelt es sich um unsere Übersetzung der diagnostischen Richtlinien - eine offizielle Übersetzung der Diagnosekriterien liegt aktuell noch nicht vor.
}

ter aggressiven Verhaltens, dessen Frequenz und Intensität außerhalb der für Alter und Entwicklungsstand erwarteten normalen Variation liegt.

Bezüglich der Verbreitung in der Bevölkerung wird angenommen, dass die intermittierende explosible Störung häufig in der späten Kindheit oder Adoleszenz einsetzt (Kröber 2015), vermehrt in stationären Populationen vorkommt (Müller et al. 2011), hohe Komorbidität (mit Persönlichkeitsstörungen) aufweist sowie mit neurologischen/ neuropsychologischen Auffälligkeiten einhergeht (Werdenich und Padlesak 2006). Trotz bereits zuvor beschriebener Ausschlusskriterien wurde eine Zwölfmonatsprävalenz der intermittierenden explosiblen Störung von ca. $4 \%$ in einer repräsentativen Stichprobe in den USA beschrieben (Kessler et al. 2006). Die hohe Komorbidität mit anderen psychischen Störungen (v.a. dissoziale und emotionalinstabile Persönlichkeitsstörung, Zwangsstörungen (Fuss et al. 2019a), aber auch psychotische und hirnorganische Störungen) legt allerdings nahe, dass es sich auch bei diesem Störungsbild wie bei der Kleptomanie und Pyromanie eher um ein Symptom verschiedener psychischer Erkrankungen statt um eine isolierte Störung aggressiver Impulse handeln dürfte. Inwieweit sich diese Auffassung bestätigt, werden kommende klinische Untersuchungen zeigen.

Insgesamt handelt es sich beim Kapitel der Impulskontrollstörungen in der ICD-11 immer noch mehr um eine Art Sammel- und Restkategorie für unterschiedliche, nicht an anderer Stelle klassifizierbare Störungsbilder, die allein aufgrund deskriptiver Ähnlichkeiten zusammengruppiert wurden. Es handelt sich um in Forschung und Klinik vernachlässigte und/oder umstrittene Diagnosen. Offene Fragen betreffen deren Legitimation als eigenständige Störungsbilder oder - aufgrund ihrer Gemeinsamkeiten sowohl mit Verhaltenssüchten als auch mit Zwangserkrankungen - deren konzeptuelle Einordnung, welche sich auf Basis der Datenlage im Verlauf verändern kann (s. am Beispiel des pathologischen Glücksspiels). Dies erklärt, dass in der Kategorie der Impulskontrollstörungen mehr Bewegung stattfindet als in anderen Kategorien und sie eine größere Angriffsfläche für Kritik und Diskussionspunkte bietet (Werdenich und Padlesak 2006, S. 541).

\section{Diskussionen rund um das Kapitel der Impulskontrollstörungen}

Diskussionspunkte zum Thema Impulskontrollstörungen können unter der Leitfrage „Ist abweichendes Sozialverhalten tatsächlich Ausdruck einer psychischen Störung?“ zusammengefasst werden. Im Kapitel der Impulskontrollstörungen würden alltägliche Verhaltensweisen als stabile Muster abweichenden Sozialverhaltens beschrieben, die erst durch ihre exzessive Ausübung abweichend wir- 
ken oder aufgrund ihrer strafrechtlichen Relevanz in den Verdacht einer Psychopathologie gerieten (Kröber 2015; Leygraf 2016). Ihr gemeinsames zentrales Merkmal, die Impulsivität als beeinträchtigte psychische Dimension, erscheint wenig spezifisch, vielmehr Symptom verschiedenster psychischer Störungen (Werdenich und Padlesak 2006). Deshalb wurde kritisiert, dass aufgrund der ungeklärten Ursachen der als Störungen der Impulskontrolle beschriebenen Verhaltensweisen nicht klar sei, ob deren Einordnung als psychische Störung in ein psychiatrisches Klassifikationssystem angemessen ist (Kröber 2015). Insgesamt werden immer wieder Stimmen mit Forderungen nach Revision einer ,wenig zufriedenstellenden Mischkategorie" (Werdenich und Padlesak 2006, S. 541) laut, denen in der ICD-11 - durch Modifikation bestehender und Aufnahme neuer Diagnosen - nur teilweise begegnet wurde.

\section{Modifikation von Diagnosen am Beispiel des pathologischen Glücksspiels}

Das pathologische Glücksspiel, ,einstiges Flaggschiff der Impulskontrollstörungen“ (Kröber 2015, S. 149), wird in der ICD-11 nicht mehr im Kapitel der Impulskontrollstörungen zu finden sein. Jahrzehntelange Kämpfe um das exzessive Glücksspielen zwischen Vertretern eines Zwangsmodelles und Suchttherapeuten (Kröber 2009, 2015) führten zunächst zu einer Kompromisskategorisierung als Störung der Impulskontrolle in der ICD-10, ebenso wie in DSM-III bis DSM-5. Forschungsbemühungen, die durch die Listung in Diagnosemanualen begünstigt wurden, resultieren nun in der ICD-11 in der Einführung einer neuen Kategorie für Verhaltenssüchte im Bereich der Suchterkrankungen (,disorders due to addictive behaviors"). Die Erweiterung des Suchtkonzepts sowie die Klassifizierung der Diagnose pathologisches Glücksspiel als Verhaltenssucht basieren auf vergleichbaren verhaltensbezogenen Symptomen von substanzgebundenen Süchten und den neuen sog. Verhaltenssüchten (Leygraf 2016), sowie auf ähnlichen neurobiologischen Korrelaten in Hirnregionen, die mit geringer Impulskontrolle, der Verarbeitung von Belohnungsreizen und aggressivem Verhalten assoziiert sind (Stieglitz und Hiller 2013).

Dieses Beispiel zeigt, dass die Aufnahme von Diagnosen in Klassifikationssysteme Forschungsaktivitäten anstoBen kann, die zur Evaluierung und Weiterentwicklung der Diagnose beitragen sowie langfristig zu einer Änderung der Konzeptualisierung führen können. Ähnliche Diskussionen finden aktuell im Bereich der zwanghaften sexuellen Verhaltensstörung statt.

\section{Einführung neuer Diagnosen am Beispiel der zwanghaften sexuellen Verhaltensstörung}

Eng verbunden mit Änderungsprozessen in Klassifikationssystemen ist die Frage nach der Notwendigkeit neuer Diagnosen, die mit dem Risiko einer ,inflationären Zunahme psychischer Störungen“ sowie einer „Trivialisierung des Konzepts psychischer Störungen" einherzugehen drohen (Stieglitz und Hiller 2013, S. 237). Dieses Spannungsverhältnis soll im Folgenden in Bezug auf die in der ICD-11 neu eingeführte und umstrittene Diagnose der zwanghaften sexuellen Verhaltensstörung diskutiert werden.

Nachdem bisherige Vorschläge zur Integration außer Kontrolle geratenen Sexualverhaltens in ein Klassifikationssystem abgelehnt wurden (z.B. hypersexuelle Störung; Kafka 2010), wird nun mit der zwanghaften sexuellen Verhaltensstörung ein jahrzehntelang diskutiertes Phänomen in die ICD-11 aufgenommen. Die gegenwärtigen Diskussionen zeigen, dass die eine Seite soziale, klinische und rechtliche Bedenken gegen die Einführung der Diagnose hat und auch dieses Symptom eher als Ausdruck anderer zugrunde liegender Störungen (z.B. Persönlichkeitsstörungen) auffasst - die andere Seite v.a. die Chance zur empirisch fundierten Weiterentwicklung des Konstrukts und zur besseren klinischen Versorgung (Fuss et al. 2019b) sieht.

Auf sozialer Ebene entsteht durch die Konzeptualisierung als psychische Störung das Risiko der Stigmatisierung (Briken 2016). Abweichendes Sexualverhalten erscheint in besonderem Maße anfällig für Stigmatisierung, weil Sexualität ohnehin mit verschiedensten Stereotypen (z. B. Herek und McLemore 2013; Boysen et al. 2014) sowie gesellschaftlichen Norm- und Wertvorstellungen (Soble 2009) konfrontiert ist. Zumal die ICD als weltweites Klassifikationssystem auch in Ländern gilt, wo die Gefahr besteht, dass eine Konzeptualisierung abweichender Sexualität als psychische Störung (religiös oder moralisch begründete) sexualfeindliche Einstellungen und die Entwicklung fragwürdiger Therapieformen begünstigen könnte (Stein et al. 2020). Die Annahme, dass die meisten TherapeutInnen und Ärzte verlässlich zwischen tatsächlich gestörtem und anderem Sexualverhalten, das lediglich aufgrund moralischer Wertungen oder der Missbilligung besonderer sexueller Verhaltensweisen gestört wirkt, unterscheiden können, ist die Hoffnung. Die Frage, ob ihnen dies gelingt, soll durch eine weltweite Fall-Kontroll-Feldstudie in Kooperation mit der Weltgesundheitsorganisation (WHO) geklärt werden, deren Datenerhebung nahezu abgeschlossen ist.

Um eine unangemessene Pathologisierung sexueller Verhaltensweisen und den missbräuchlichen Gebrauch der Diagnose weniger wahrscheinlich zu machen, soll die zwanghafte sexuelle Verhaltensstörung nicht diagnostiziert werden, wenn ein hohes Maß an sexuellem Interesse und sexu- 
ellem Verhalten, jedoch ohne Leidensdruck oder eine Beeinträchtigung der sexuellen Selbstkontrolle vorliegt. Ebenso wenig, wenn das Verhalten nur über einen kurzen Zeitraum oder bei Jugendlichen auftritt. Im klinischen Kontext befürchten KritikerInnen, dass durch Pathologisierung sexueller Normvarianten (Fuss et al. 2018; Klein et al. 2019) ein hohes Potenzial für Falsch-positiv-Raten entsteht (Winters 2010; Steele et al. 2013). Daher sollen die neuen Diagnosekriterien - im Vergleich zum bloßen Vorhandensein einer diagnostischen Kategorie ohne weitere Spezifikationen wie in der ICD-10 - dazu beitragen, dass die Schwelle für die Diagnose einerseits nicht zu niedrig ist, andererseits keine zu hohe Hürde für eine mögliche Behandlung betroffener Personen darstellt. Besonders wichtig erscheint dabei die Differenzierung zwischen Personen, die tatsächlich unter ihrem Sexualverhalten leiden, und solchen, die Konzepte wie „Sexsucht“ beispielsweise als Rechtfertigung für Fehlverhalten nutzen (Kraus et al. 2016, 2018; Gola und Potenza 2018). In diesem Zusammenhang wurden auch rechtliche Bedenken wie eine Fehlanwendung der Diagnose vor Gericht diskutiert (Kafka 2010, 2014). Trotz Fehlens einer offiziellen Diagnose fand das verwandte Konstrukt ,sex addiction" v.a. in amerikanischen Gerichtsverfahren bereits Anwendung (Ley et al. 2015 für Fallbeispiele). KritikerInnen befürchten, dass eine offizielle Diagnose insbesondere im Zusammenhang mit Sexualstraftaten im angloamerikanischen Rechtssystem als strafmildernder Faktor missbraucht werden könnte (Ley et al. 2015; Montgomery-Graham 2017).

Weitere Kritikpunkte betreffen die mangelnde empirische Fundierung des Konzepts der zwanghaften sexuellen Verhaltensstörung, ihre konzeptuelle Einordnung, ihre Diagnosekriterien sowie mögliche Therapieansätze (Stein et al. 2020). Besonders die konzeptuelle Einordnung als Störung der Impulskontrolle sowie alternative Konzeptualisierungsvorschläge als Verhaltenssucht oder Zwangsstörung wurden unter öffentlichen, politischen und finanziellen Gesichtspunkten kontrovers diskutiert (Böthe et al. 2018a, b; Carnes 1991; Fuss et al. 2019a, b; Gola und Potenza 2018; Grant et al. 2014; Griffiths 2016; Kraus et al. 2016; Potenza et al. 2017; Stein 2008; Stein et al. 2020).

Mit der Aufnahme der zwanghaften sexuellen Verhaltensstörung in die ICD-11 wird die jahrzehntelange Debatte um das Phänomen keineswegs beendet sein, der Teufelskreis aus fehlenden offiziellen Diagnosekriterien und fehlender empirischer Fundierung aber allemal beendet. Als Gegenstand aktueller und künftiger Forschungsbestrebungen hat die neue Diagnose das Potenzial, Veränderungen in Therapie und Prävention anzustoßen (Mead und Sharpe 2018). Inwiefern die Diagnose die soziale, klinische und rechtliche Situation tatsächlich verändert, wird sich im Anschluss an ihre Einführung zeigen. Mögliche Implikationen werden im nächsten Abschnitt dargestellt.

\section{Implikationen}

Beschriebenen Risiken der Weiterentwicklung bestehender Diagnosen und Aufnahme neuer Diagnosen in Klassifikationssysteme (Diagnoseinflation, Trivialisierung psychischer Störungen, Pathologisierung abweichenden Verhaltens, hohe Falsch-positiv-Raten, Missbrauch von Diagnosen) stehen wichtige Chancen für die Forschung und klinisch-therapeutische Praxis gegenüber. Im Folgenden werden wissenschaftliche, klinische und rechtliche Implikationen dargestellt, die mit den ICD-11-Neuerungen im Kapitel der Impulskontrollstörungen einhergehen.

\section{Implikationen für die Forschung}

Das Beispiel der zwanghaften sexuellen Verhaltensstörung zeigt, wie Forschungsbestrebungen durch die Einführung neuer Diagnosen angeregt werden können. Seit Konzeptualisierung als Impulskontrollstörung ist die Anzahl an Studien zum Thema zwanghafte Sexualität stark angewachsen. Unter anderem, wurde bereits ein Messinstrument anhand der ICD-11-Kriterien entwickelt und in verschiedenen Sprachen validiert (Böthe et al. 2020); Prävalenzschätzungen sowie ätiologische (Leeman et al. 2019; Walton et al. 2017) und Untersuchungen zu Therapieansätzen wurden durchgeführt (Hallberg et al. 2019). Allgemein haben wissenschaftliche Erkenntnisse auf Basis valider und reliabler Diagnosekriterien die Bedeutung von Impulskontrollstörungen deutlicher gemacht. Vor allem die zwanghafte sexuelle Verhaltensstörung, das pathologische Glücksspiel und die intermittierende explosible Störung scheinen in der Allgemeinbevölkerung verbreitete Erkrankungen, die mit Beeinträchtigungen in verschiedenen Lebensbereichen einhergehen und die Wichtigkeit wissenschaftlich fundierter Diagnostik sowie Behandlung betonen (Grant et al. 2014). Aus dieser Perspektive ist deren Konzeptualisierung als Impulskontrollstörung auch bei strittiger Datenlage eher zu begrüßen.

\section{Klinische Implikationen}

Klare diagnostische Richtlinien und eine empirische Fundierung von Störungskonzepten können auf klinischer Ebene zur Verbesserung der Behandlungssituation beitragen. Die Listung in der ICD-11 scheint die Behandlungsmöglichkeiten zu verbessern und die Chancen zu erhöhen, dass Behandlungskosten durch Krankenkassen übernommen werden können (Stein et al. 2020). Auch können dadurch TherapeutInnen für das jeweilige Krankheitsbild sensibilisiert, der Einfluss moralischer, religiöser oder sonstiger Urteile verringert und der Spielraum für unseriöse Therapieangebote (z.B. „Suchtrehabilitation“ für „Sexsucht“) eingeschränkt werden (Gola und Potenza 2018). Damit 
einher geht im besten Fall die Entwicklung und wissenschaftliche Überprüfung spezifischer Therapieprogramme.

Dass es sich bei den Impulskontrollstörungen um eine Gruppe im Grundsatz normaler Verhaltensvarianten handelt, macht die Diagnostik der Störungen zu einer anspruchsvollen Gratwanderung. Umso wichtiger sind explizite Diagnosekriterien (wie für die zwanghafte sexuelle Verhaltensstörung in der ICD-11, inklusive Kriterien zur Abgrenzung von Normvarianten sexuellen Verhaltens und anderen psychischen Störungen), anhand derer eine Differenzierung zwischen normalem und pathologischem Verhalten möglich sein soll. Vor allem, wenn die Behandlungsmotivation ggf. auf geschlechtstypischen Erwartungshaltungen, sexualitätsfeindlichen Einstellungen oder überhöhten Moralvorstellungen (Klein et al. 2015) basiert, ist eine Abgrenzung schwierig; aber wichtig, um negative Folgen für Betroffene (z.B. Stigmatisierung, Medikalisierung sexuellen Verhaltens, nichtindizierte Pharmakotherapien, inklusive Nebenwirkungen) und das Gesundheitssystem (z.B. Verknappung der Behandlungsressourcen) zu vermeiden (Briken 2016). Eine wichtige Rolle kommt hierbei einem gemeinsamen Kernmerkmal der Impulskontrollstörungen zu: Leidensdruck und Beeinträchtigungen in verschiedenen Funktionsbereichen sollen nicht ausschließlich auf sozialen, moralischen oder sonstigen Wertungen basieren. Störungsübergreifend und unabhängig von der konzeptuellen Einordnung der Störung sollen darüber hinaus die Motivlage und Funktion des impulsiven Verhaltens (z.B. exzessives Sexualverhalten, Brandstiftung, Glücksspiel) im therapeutischen Kontext analysiert werden: Werden die Verhaltensweisen primär zur Bewältigung innerer Spannungszustände und zum Abbau unangenehmer Gefühle durchgeführt oder wird ein bestimmter emotionaler Zustand (z.B. Lustgewinn, euphorische Stimmungslage) angestrebt (Briken 2016; Werdenich und Padlesak 2006)? Dass die Konzeptualisierung von Impulskontrollstörungen - obwohl ähnlich kontrovers diskutiert wie die Diagnosekriterien - im therapeutischen Kontext eine untergeordnete Rolle spielt, zeigt sich am Beispiel des pathologischen Glücksspiels. Unabhängig von der Einordnung der Störung als Impulskontrollstörung (ICD-10) oder Verhaltenssucht (ICD-11) kommt eine Reihe von Behandlungsmöglichkeiten infrage, die weder dem einen noch dem anderen Bereich entsprungen sind (z. B. Lithium- oder Expositionstherapie; Cowlishaw et al. 2012; Hollander et al. 2005). Ein ähnliches therapeutisches Vorgehen trotz Umkategorisierung des pathologischen Glücksspiels sowie eine Verbesserung der Versorgungslage durch die neue Diagnose zwanghafte sexuelle Verhaltensstörung in der ICD-11 können zumindest auf klinischer Ebene einige der am Kapitel der Impulskontrollstörungen geäußerten Kritikpunkte relativieren.

\section{Rechtliche Implikationen}

Das Kapitel der Impulskontrollstörungen erscheint besonders im Hinblick auf forensisch-psychiatrische Fragestellungen relevant, weil alle beschriebenen Verhaltensweisen strafrechtlich bedeutsam sein können (z.B. in Form von Brandstiftung, Diebstahl, aggressiven Ausbrüchen mit Körperverletzung, Sexualdelikten). Unvorbereitet impulsives Verhalten kann ein Hinweis auf eine Beeinträchtigung der exekutiven Steuerungsfähigkeit sein, weshalb den Diagnosen eine zentrale Bedeutung im Kontext der Beurteilung von Steuerungsfähigkeit und Schuldfähigkeit zukommen könnte.

Die Frage nach verminderter oder aufgehobener Schuldfähigkeit bei Straftaten kommt auf, wenn ein Zusammenhang zwischen der Entstehung eines Delikts und Symptomen einer psychischen Störung angenommen wird. Im Rahmen einer gerichtlich in Auftrag gegebenen forensischpsychiatrischen Begutachtung wird untersucht, ob pathologisches Verhalten im Sinne einer psychischen Störung vorliegt, die die Voraussetzungen eines von vier vom Gesetzgeber vorgegebenen Kriterien, unter denen die Schuldfähigkeit gemindert oder aufgehoben sein kann ( $\S 20,21$ StGB), erfüllt. Eine Einschränkung der strafrechtlichen Verantwortlichkeit aufgrund einer psychischen Störung kommt in Betracht, wenn die diagnostizierte Störung einen Zusammenhang zum delinquenten Verhalten aufweist. Störungen der Impulskontrolle können wie Persönlichkeitsstörungen oder Paraphilien unter das 4. Kriterium der „schweren anderen seelischen Abartigkeit" (SASA) fallen und damit die Frage einer erheblichen Verminderung der Steuerungsfähigkeit zur Diskussion stellen.

Die forensisch-psychiatrische Begutachtung im Zusammenhang mit Impulskontrollstörungen scheint anspruchsvoll zu sein, weil delinquente Verhaltensweisen (z. B. Diebstahl, Gewalttaten) als solche in den Diagnosekriterien enthalten sind (Kröber 2015). Doch laut Ausschlusskriterien der Impulskontrollstörungen in der ICD-11 handelt es sich bei den Diagnosen um störungsbedingte Einbußen im Symptombereich der Impulskontrolle, die nicht durch eine andere psychische Störung erklärt werden können. Ohne das Vorliegen weiterer Komorbiditäten dürften diese störungsbedingten Einbußen im Regelfall nicht zu einer schwerwiegenden Beeinträchtigung der Beziehungsgestaltung und psychosozialen Leistungsfähigkeit führen. Isolierte Funktionsstörungen im Bereich der Impulskontrolle als eigenständige Störungsbilder dürften in Ausgestaltung und Schweregrad dementsprechend nicht die forensisch-psychiatrischen Voraussetzungen für eine SASA erfüllen.

Geht man hingegen von einer komorbiden Störung (wie z.B. einer Persönlichkeitsstörung) aus, die den Schweregrad einer SASA erreicht, so kann der jeweiligen Impulskontrollstörung in Ausnahmefällen eine bedeutsame Rolle 
zukommen. Beispielsweise wenn es schwierig ist, das strafrechtlich relevante Verhalten im Zusammenhang mit der Persönlichkeitsstörung psychopathologisch einzuordnen. Denkbar ist, dass dann eine scheinbar motivlose Impulskontrollstörung als Verstehenshintergrund für das Verhalten herangezogen wird. In solch einem Fall sollte bedacht werden, dass eine entsprechende Anwendung der Diagnosen mit weitreichenden Konsequenzen - von Strafminderung bis zur zeitlich unbegrenzten Unterbringung in einem psychiatrischen Krankenhaus nach $\S 63$ StGB - verbunden sein kann. Zum Beispiel kann die Diagnose einer zwanghaften sexuellen Verhaltensstörung im Zusammenhang mit Sexualdelinquenz ähnlich wie eine paraphile Störung zu einer negativen Kriminalprognose und zu einer Unterbringung im Maßregelvollzug beitragen. Allerdings hat zwanghaftes Sexualverhalten durch das Konstrukt sexueller Süchtigkeit (Giese 1962) bereits zuvor, unabhängig von der neuen Diagnose der zwanghaften sexuellen Verhaltensstörung, eine Rolle in der Schuldfähigkeitsbegutachtung gespielt (Briken 2016) und dürfte dahingehend keine bedeutenden Änderungen anstoßen.

Insgesamt sollten Impulskontrollstörungen im forensisch-psychiatrischen Kontext hauptsächlich dahingehend eine Rolle spielen, dass Impulskontrolle generell als prognostisch relevanter Symptombereich im Begutachtungsprozess beachtet werden sollte. Die Diagnose einer isolierten Impulskontrollstörung dürfte in der forensischen Praxis allerdings nur in den seltensten Fällen aufgrund oben genannter Gründe als Hinweis auf eine Minderung der Steuerungs- und Schuldfähigkeit gewertet werden. Die wichtigste Rolle kommt unabhängig von der Diagnosestellung nach wie vor der psychopathologischen Analyse von Diagnose und Delikt sowie der Expertise des Gutachters zu. Demnach wird sich die forensische Praxis durch die Impulskontrollstörungen der ICD-11 vermutlich kaum ändern, wahrscheinlich aber vom verbesserten Verständnis der Störungsbilder langfristig profitieren, das sich aus verstärkter Forschungsaktivität ergeben könnte. So wurde jüngst beispielsweise die wichtige Unterscheidung zwischen einem empfundenen Kontrollverlust über sexuelles Verhalten entsprechend den Diagnosekriterien und einer forensisch-relevanten Steuerungsminderung betont (Fuss et al. 2020) oder das Konzept der sexuellen Dranghaftigkeit im Rahmen der Schuldfähigkeitsbegutachtung neu diskutiert (Briken 2016).

\section{Fazit}

Nach teils kontroversen Diskussionen um das Konzept der Impulskontrollstörungen i.Allg. und einzelne Diagnosen entschied die Weltgesundheitsorganisation (WHO) zugunsten der Impulskontrollstörungen als weiterhin ei- gene Störungskategorie in der International Classification of Diseases and Related Health Problems (ICD-11), inklusive der Aufnahme der neuen Diagnosen zwanghafte sexuelle Verhaltensstörung und intermittierende explosible Störung. Die Aufnahme weiterer vermeintlicher Impulskontrollstörungen wie problematische Internetnutzung oder zwanghaftes Kaufen wurde aufgrund der unzureichenden Datenlage zum gegenwärtigen Zeitpunkt abgelehnt. Eine fehlende empirische Fundierung wurde neben sozialen (z.B. Stigmatisierung), klinischen (z.B. Pathologisierung sexueller Normvarianten) und rechtlichen (z. B. Missbrauch der Diagnose als Rechtfertigung für Sexualstraftaten) Bedenken auch als Hauptargument gegen eine Diagnose für zwanghaftes Sexualverhalten angeführt. Einige dieser Kritikpunkte am Kapitel der Impulskontrollstörungen können durch Vorteile in der Forschung (z.B. ätiologische und Prävalenzstudien) und Therapie (z.B. Behandlungskostenübernahme, Sensibilisierung von TherapeutInnen) relativiert werden. Trotz unterschiedlicher bzw. unbekannter Ätiologie der enthaltenen Störungsbilder können deskriptive Ähnlichkeiten in den Symptomen (z.B. Unkontrollierbarkeit von Impulsen, anfänglicher Belohnungscharakter, langfristig Beeinträchtigungen in wichtigen Lebensbereichen, Leidensdruck) als Anhaltspunkte für die Behandlung von Impulskontrollstörungen genutzt werden. Konzeptuelle Diskussionen scheinen für die klinisch-therapeutische Praxis eine untergeordnete Rolle zu spielen. Gründliche Begutachtungsprozesse können dem Missbrauch psychischer Störung im rechtlichen Kontext entgegenwirken. Wenn man die Änderungen der ICD-11 als aktuellen psychiatrischen Forschungs- und Erfahrungsstand, nicht wie starre Richtlinien, versteht und unter Beachtung potenzieller Schwächen anwendet, können sie neue Impulse für die ständige Weiterentwicklung und Verbesserung der Klassifikation und Behandlung psychischer Störungen beitragen.

Funding Open Access funding enabled and organized by Projekt DEAL.

Interessenkonflikt S. Bründl und J. Fuss geben an, dass kein Interessenkonflikt besteht.

Open Access Dieser Artikel wird unter der Creative Commons Namensnennung 4.0 International Lizenz veröffentlicht, welche die Nutzung, Vervielfältigung, Bearbeitung, Verbreitung und Wiedergabe in jeglichem Medium und Format erlaubt, sofern Sie den/die ursprünglichen Autor(en) und die Quelle ordnungsgemäß nennen, einen Link zur Creative Commons Lizenz beifügen und angeben, ob Änderungen vorgenommen wurden.

Die in diesem Artikel enthaltenen Bilder und sonstiges Drittmaterial unterliegen ebenfalls der genannten Creative Commons Lizenz, sofern sich aus der Abbildungslegende nichts anderes ergibt. Sofern das betreffende Material nicht unter der genannten Creative Commons Lizenz steht und die betreffende Handlung nicht nach gesetzlichen Vorschriften erlaubt ist, ist für die oben aufgeführten Weiterverwendungen des Materials die Einwilligung des jeweiligen Rechteinhabers einzuholen. 
Weitere Details zur Lizenz entnehmen Sie bitte der Lizenzinformation auf http://creativecommons.org/licenses/by/4.0/deed.de.

\section{Literatur}

APA (American Psychiatric Association) (2013) Diagnostic and statistical manual of mental disorders (DSM-5). American Psychiatric Publishing, Arlington

Barnett W (2005) Psychiatrie der Brandstiftung: eine psychopathologische Studie anhand von Gutachten Bd. 110. Steinkopff, Darmstadt

Boysen G, Ebersole A, Casner R, Coston N (2014) Gendered mental disorders: masculine and feminine stereotypes about mental disorders and their relation to stigma. J Soc Psychol 154(6):546-565

Briken P (2016) Das Konstrukt „,sexuelle Sucht“ im Zusammenhang mit forensisch psychiatrischen Fragestellungen. Forens Psychiatr Psychol Kriminol 10(3):173-180

Burton PR, McNiel DE, Binder RL (2012) Firesetting, arson, pyromania, and the forensic mental health expert. J Am Acad Psychiatr Law Online 40(3):355-365

Böthe B, Bartók R, Tóth-Király I, Reid RC, Griffiths MD, Demetrovics $Z$, Orosz G (2018a) Hypersexuality, gender, and sexual orientation: a large-scale psychometric survey study. Arch Sex Behav 47(8):2265-2276

Böthe B, Tóth-Király I, Potenza MN, Griffiths MD, Orosz G, Demetrovics $Z$ (2018b) Revisiting the role of impulsivity and compulsivity in problematic sexual behaviors. J Sex Res 56(2):166-179

Böthe B, Potenza MN, Griffiths MD, Kraus SW, Klein V, Fuss J, Demetrovics Z (2020) The development of the compulsive sexual behavior disorder scale (CSBD-19): an ICD-11 based screening measure across three languages. J Behav Addict 9(2):247-258

Carnes P (1991) Don't call it love: recovery from sexual addiction. Bantam, New York

Coleman E (1991) Compulsive sexual behavior. J Psychol Human Sex 4(2):37-52

Cowlishaw S, Merkouris S, Dowling N, Anderson C, Jackson A, Thomas S (2012) Psychological therapies for pathological and problem gambling. Cochrane Database Syst Rev 11:CD8937

Dickenson JA, Gleason N, Coleman E, Miner MH (2018) Prevalence of distress associated with difficulty controlling sexual urges, feelings, and behaviors in the United States. JAMA Netw Open 1(7):e184468-e184468

Dilling H, Mombour W, Schmidt MH (Hrsg) (1991) Internationale Klassifikation psychischer Störungen, ICD-10, Kapitel V (F). Klinische Beschreibungen und diagnostische Leitlinien. Huber, Bern

Esquirol É (1838) Des maladies mentales. JB Baillière

Fuss J, Briken P, Klein V (2018) Gender bias in clinicians' pathologization of atypical sexuality: a randomized controlled trial with mental health professionals. Sci Rep 8:3715

Fuss J, Briken P, Stein DJ, Lochner C (2019a) Compulsive sexual behavior disorder in obsessive-compulsive disorder: prevalence and associated comorbidity. J Behav Addict 8(2):242-248

Fuss J, Lemay K, Stein DJ, Briken P, Jakob R, Reed GM, Kogan CS (2019b) Public stakeholders' comments on ICD-11 chapters related to mental and sexual health. World Psychiatry 18(2):233

Fuss J, Voulgaris A, Briken P (2020) Schuldfähigkeit bei Personen, die wegen Nutzung von Missbrauchsabbildungen angeklagt sind. Forens Psychiatr Psychol Kriminol 14:437-445

Giese H (1962) Leitsymptome sexueller Perversionen. In: Giese H (Hrsg) Psychopathologie der Sexualität. Enke, Stuttgart, S $420-470$

Gola M, Potenza MN (2018) The proof of the pudding is in the tasting: Data are needed to test models and hypotheses related to compulsive sexual behaviors. Arch Sex Behav 47(5):1323-1325

Grant JE, Atmaca M, Fineberg NA, Fontenelle LF, Matsunaga H, Reddy YJ et al (2014) Impulse control disorders and "behavioural addictions" in the ICD-11. World Psychiatry 13(2):125
Griffiths MD (2012) Internet sex addiction: a review of empirical research. Addict Res Theory 20(2):111-124

Griffiths MD (2016) Compulsive sexual behaviour as a behavioural addiction: the impact of the Internet and other issues. Addiction 111(12):2107-2108

Hallberg J, Kaldo V, Arver S, Dhejne C, Jokinen J, Öberg KG (2019) A randomized controlled study of group-administered cognitive behavioral therapy for hypersexual disorder in men. J Sex Med 16(5):733-745

Herek GM, McLemore KA (2013) Sexual prejudice. Annu Rev Psychol 64:309-333

Hollander E, Pallanti S, Allen A, Sood E, Rossi NB (2005) Does sustained-release lithium reduce impulsive gambling and affective instability versus placebo in pathological gamblers with bipolar spectrum disorders? Am J Psychiatry 162(1):137-145

Kafka MP (2010) Hypersexual disorder: a proposed diagnosis for DSM-V. Arch Sex Behav 39(2):377-400

Kafka MP (2014) What happened to hypersexual disorder? Arch Sex Behav 43(7):1259-1261

Kessler RC, Coccaro EF, Fava M, Jaeger S, Jin R, Walters E (2006) The prevalence and correlates of DSM-IV intermittent explosive disorder in the National Comorbidity Survey Replication. Arch Gen Psychiatry 63(6):669-678

Klein V, Briken P, Schröder J, Fuss J (2019) Mental health professionals' pathologization of compulsive sexual behavior: Does clients' gender and sexual orientation matter? J Abnorm Psychol 128(5):465

Klein V, Brunner F, Nieder TO, Reed G, Briken P (2015) Diagnoseleitlinien sexueller Störungen in der International Classification of Diseases and Related Health Problems (ICD)-11: Dokumentation des Revisionsprozesses. Z Sex-Forsch 28:363-373

Klein V, Rettenberger M, Briken P (2014) Self-reported indicators of hypersexuality and its correlates in a female online sample. J Sex Med 11(8):1974-1981

Krafft-Ebing R von (1903) Psychopathia sexualis, 12. Aufl. Enke, Stuttgart

Kraus SW, Krueger RB, Briken P, First MB, Stein DJ, Kaplan MS et al (2018) Compulsive sexual behaviour disorder in the ICD-11. World Psychiatry 17(1):109-110

Kraus SW, Voon V, Potenza MN (2016) Should compulsive sexual behavior be considered an addiction? Addiction 111(12):2097-2106

Kröber HL (2009) Pathologisches Glücksspielen: Persönlichkeitsmerkmale und forensische Aspekte. Forens Psychiatr Psychol Kriminol 3:90-98

Kröber HL (2015) Disruptive, Impulskontroll- und Sozialverhaltensstörungen im DSM-5. Forens Psychiatr Psychol Kriminol 9:147-154

Leeman RF, Rowland BHP, Gebru NM, Potenza MN (2019) Relationships among impulsive, addictive and sexual tendencies and behaviours: a systematic review of experimental and prospective studies in humans. Philos Trans R Soc Lond B Biol Sci 374(1766):20180129

Ley D, Brovko JM, Reid RC (2015) Forensic applications of "sex addiction" in US legal proceedings. Curr Sex Health Rep 7(2):108-116

Leygraf N (2009) Kleptomanie und Ladendiebstahl: Ein historischer Überblick. Forens Psychiatr Psychol Kriminol 3(2):107-115

Leygraf N (2016) Impulskontrollstörungen/Verhaltenssüchte. Forens Psychiatr Psychol Kriminol 10:153-154

Marc CC (1844) Die Geisteskrankheiten in Beziehung zur Rechtspflege: ein Handbuch für Gerichtsärzte und Juristen Bd. 2. Voss, Berlin

Mead D, Sharpe M (2018) Pornography and sexuality research papers at the 5th International Conference on Behavioral Addictions. Sex Addict Compulsivity 25(4):248-268

Montgomery-Graham S (2017) Disorder in the court: the approach to sex addiction in Canadian legal proceedings. Can J Hum Sex 26(3):205-215 
Müller A, Rein K, Kollei I, Rotter A, Schütz P, Hillemacher T, de Zwaan M (2011) Häufigkeit von Impulskontrollstörungen bei stationären Patienten. Psychother Psychosom Med Psychol 61(02):A56

Potenza MN, Gola M, Voon V, Kor A, Kraus SW (2017) Is excessive sexual behaviour an addictive disorder? Lancet Psychiatry 4(9):663-664

Soble A (2009) The philosophy of sexuality. The philosophy of sexuality, from the Internet encyclopedia of philosophy. https://ssrn. com/abstract=2327398. Zugegriffen: 22 . Apr. 2020

Steele VR, Staley C, Fong T, Prause N (2013) Sexual desire, not hypersexuality, is related to neurophysiological responses elicited by sexual images. Socioaffect Neurosci Psychol 3(1):20770

Stein DJ (2008) Classifying hypersexual disorders: compulsive, impulsive, and addictive models. Psychiatr Clin North Am 31(4):587-591

Stein DJ, Szatmari P, Gaebel W, Berk M, Vieta E, Maj M (2020) Mental, behavioral and neurodevelopmental disorders in the ICD-11: an international perspective on key changes and controversies. BMC Med 18(1):1-24

Stieglitz RD, Hiller W (2013) Definition und Erfassung psychischer Störungen. Psychotherapeut 58:237-248

Torales J, González I, Castaldelli-Maia JM, Ventriglio A (2020) Kleptomania as a neglected disorder in psychiatry. Int Rev Psychiatry 32(5-6):451-454

Walton MT, Cantor JM, Bhullar N, Lykins AD (2017) Hypersexuality: a critical review and introduction to the "sexhavior cycle". Arch Sex Behav 46:2231-2251

Werdenich W, Padlesak S (2006) 〈F63〉 Abnorme Gewohnheiten und Störungen der Impulskontrolle. In: Handbuch der klinisch-psychologischen Behandlung. Springer, Wien, S 541-558

Winters J (2010) Hypersexual disorder: a more cautious approach. Arch Sex Behav 39(3):594-596 